\title{
Mass Determination of Groups of Galaxies: Effects of the Cosmological Constant
}

\author{
S. Peirani* and J.A. de Freitas Pacheco \\ Observatoire de la Côte d'Azur, B.P. 4229, F-06304 Nice Cedex 4, France
}

\begin{abstract}
The spherical infall model first developed by Lemaitre and Tolman was modified in order to include the effects of a dark energy term. The resulting velocity-distance relation was evaluated numerically. This equation, when fitted to actual data, permits the simultaneous evaluation of the central mass and of the Hubble parameter. Application of this relation to the Local Group, when the dark energy is modeled by a cosmological constant, yields a total mass for the M31-Milky Way pair of $(2.5 \pm 0.7) \times 10^{12} M_{\odot}$, a Hubble parameter $\mathrm{H}_{0}=74 \pm 4 \mathrm{kms}^{-1} \mathrm{Mpc}^{-1}$ and a 1 -D velocity dispersion for the flow of about $39 \mathrm{kms}^{-1}$. The zero-velocity and the marginally bound surfaces of the Local Group are at about 1.0 and $2.3 \mathrm{Mpc}$ respectively from the center of mass. A similar analysis for the Virgo cluster yields a mass of $(1.10 \pm 0.12) \times 10^{15} M_{\odot}$ and $\mathrm{H}_{0}=65 \pm 9 \mathrm{kms}^{-1} \mathrm{Mpc}^{-1}$. The zero-velocity is located at a distance of $8.6 \pm 0.8 \mathrm{Mpc}$ from the center of the cluster. The predicted peculiar velocity of the Local Group towards Virgo is about $190 \mathrm{kms}^{-1}$, in agreement with other estimates. Slightly lower masses are derived if the dark energy is represented by a fluid with an equation of state $P=w \epsilon$ with $w=-2 / 3$.
\end{abstract}

Key words: Local Group, Virgo Cluster, Hubble constant

PACS: 98.62.Ck, 98.65.Bv, 98.65.Cw

\section{Introduction}

New and high quality data on galaxies belonging to nearby groups have improved considerably estimates of masses and mass-to-light ratios (M/L) of

* Corresponding author.

Email addresses: peirani@obs-nice.fr (S. Peirani), pacheco@obs-nice.fr (J.A. de Freitas Pacheco). 
these systems. Searches on the POSS II and ESO/SERC plates (Karachentseva \& Karachentsev 1998; 2000) as well as "blind" HI surveys (Kilborn et al. 2002) lead to the discovery of new dwarf galaxies, increasing substantially their known population in the local universe. Moreover, in the past years, using HST observations, distances to individual members of nearby groups have been derived from magnitudes of the tip of the red giant branch by Karachentsev and collaborators (Karachentsev 2005 and references therein), which have permitted a better membership assignment and a more trustful dynamical analysis.

Previous estimates of $M / L$ ratios for nearby groups were around $170 M_{\odot} / L_{B, \odot}$ (Huchra \& Geller 1982). However, virial masses derived from the aforementioned data are significantly smaller, yielding $M / L$ ratios around $10-30 M_{\odot} / L_{B, \odot}$. If these values are correct, the local matter density derived from nearby groups would be only a fraction of the global matter density (Karachentsev 2005). However, for several groups the crossing time is comparable or even greater than the Hubble time and another approach is necessary to evaluate their masses, since dynamical equilibrium is not yet attained in these cases.

Lynden-Bell (1981) and Sandage (1986) proposed an alternative method to the virial relation in order to estimate the mass of the Local Group, which can be extended to other systems dominated either by one or a pair of galaxies. Their analysis is essentially based on the spherical infall model. If the motion of bound satellites is supposed to be radial, the resulting parametric equations describe a cycloid. Initially, the radius of a given shell embedding a total mass $\mathrm{M}$ expands, attains a maximum value and then collapses. At maximum, when the turnaround radius $R_{0}$ is reached, the radial velocity with respect to the center of mass is zero. For a given group, if the velocity field close to the main body, probed by satellites, allows the determination of $\mathrm{R}_{0}$, then the mass can be calculated straightforwardly from the relation

$$
M=\frac{\pi^{2} R_{0}^{3}}{8 G T_{0}^{2}}
$$

where $T_{0}$ is the age of the universe and $G$ is the gravitational constant.

Data on the angular power spectrum of temperature fluctuations of the cosmic microwave background radiation derived by WMAP (Spergel et al. 2003) and on the luminosity-distance of type Ia supernovae (Riess et al. 1998; Perlmutter et al. 1999), lead to the so-called "concordant" model, e.g., a flat cosmological model in which $\Omega_{m}=0.3$ and $\Omega_{v}=0.7$. The later density parameter corresponds to the present contribution of a cosmological constant term or a fluid with negative pressure, dubbed "quintessence" or dark energy. The radial motion leading to the aforementioned $M=M\left(R_{0}, T_{0}\right)$ relation neglects the effect of such a term, which acts as a "repulsive" force. This repulsive 
force is proportional to the distance and its effect can be neglected if the zerovelocity surface is close to the center of mass. Turnaround radii of groups are typically of the order of $1 \mathrm{Mpc}$ (Karachentsev 2005), while the characteristic radius at which gravitation is comparable to the repulsion force is $\mathrm{R}_{*}=$ $1.1 \mathrm{M}_{12}^{1 / 3} \mathrm{Mpc}$, where $\mathrm{M}_{12}=\mathrm{M} /\left(10^{12} M_{\odot}\right)$ and the Hubble parameter $H_{0}$ was taken equal to $70 \mathrm{kms}^{-1} \mathrm{Mpc}^{-1}$. This simple argument suggests that the effect of the cosmological term can not be neglected when deriving masses from the $M=M\left(R_{0}, T_{0}\right)$ relationship.

In this paper we revisit the velocity-distance relationship when a dark energy term is included in the dynamical equations and calculate the resulting $M=M\left(R_{0}, H_{0}\right)$ relation. The presence of the dark energy term has been also invoked as a possible explanation for the smoothness of the local Hubble flow (Chernin 2001; Teerikorpi, Chernin and Baryshev 2005) being a further reason to investigate its effects on the $M=M\left(R_{0}, T_{0}\right)$ relation. For a cosmological density parameter associated to the "vacuum" energy $\Omega_{v}=0.7$ (our preferred solution), numerical computations indicate that the "zero-energy" surface, beyond which galaxies will never collapse onto the core, is located at about $2.3 R_{0}$. In order to illustrate our results, some applications are made to the Local Group and the Virgo cluster. As we shall see, values of the Hubble parameter resulting from fits of the actual data to the velocity-distance relation including a cosmological term, are in better agreement with recent estimates then those derived from the relation obtained either by Lyndell-Bell (1981) or Sandage (1986). In Section 2 the relevant equations are introduced, in Sections 3 and 4 the results are applied to the Local Group and the Virgo cluster and finally, in Section 5 the concluding remarks are given.

\section{The velocity-distance relation}

The evolution of a self-gravitating zero-pressure fluid with spherical symmetry was first considered by Lemaitre (1933) and Tolman (1934). The LemaitreTolman model describes quite well the dynamics of an extended halo around a bound central core, asymptotically approaching a homogeneous Friedmann background. In this situation, three main distinct regions can be distinguished: i) the central core, in which the shell crossing has already occurred, leading to energy exchanges which transform radial into transverse motion; ii) the zerovelocity surface, boundary which separates infalling and expanding bound shells and iii) the "marginally" bound surface (zero total energy), segregating bound and unbound shells. Density profiles resulting from the LemaitreTolman model were examined by Olson \& Silk (1979) and application of this model to the velocity field close to the Virgo cluster were made by Hoffman et al. (1980), Tully \& Shaya (1984), Teerikorpi et al. (1992) among others. 
If displacements of galaxies, here associated to the outer halo shells, develop mainly at low redhsifts when the formation of the mass concentration around the core is nearly complete (see, for instance, Peebles 1990), then the equation of motion for a spherical shell of mass $m$, moving radially in the gravitational field created by a mass $M$ inside a shell of radius $\mathrm{R}$, including the dark energy term is

$$
\frac{d^{2} R}{d t^{2}}=-\frac{G M}{R^{2}}-\frac{(1+3 w)}{2} \Omega_{v} H_{0}^{2} R\left(\frac{a_{0}}{a}\right)^{3(1+w)}
$$

where $M=4 \pi \int_{0}^{R} r^{2} \rho_{m} d r$ and $a$ is the scale parameter (the present value is taken as $a_{0}=1$ ). The latter satisfies the Hubble equation

$$
\left(\frac{d l g a}{d t}\right)^{2}=H_{0}\left[\Omega_{m}\left(\frac{a_{0}}{a}\right)^{3}+\Omega_{v}\left(\frac{a_{0}}{a}\right)^{3(1+w)}\right]
$$

Here, the common assumption that the dark energy can be modeled as being a fluid with an equation of state $P=w \epsilon$ was adopted and in the two equations above, the dependence of the dark energy on the scale parameter was obtained by solving the energy conservation for such a component. Eq. (2) is intended to describe the motion of shells in the halo, excluding the central region where shell crossing effects have probably already occurred.

Defining the dimensionless variables $y=R / R_{0}, \tau=t H_{0}$ and $x=a / a_{0}$, eqs. (2) and (3) can be rewritten as

$$
\frac{d^{2} y}{d \tau^{2}}=-\frac{1}{2}\left[\frac{A}{y^{2}}+B y x^{-3(1+w)}\right]
$$

and

$$
\frac{d x}{d \tau}=\sqrt{\frac{\Omega_{m}}{x}+\frac{\Omega_{v}}{x^{(1+3 w)}}}
$$

where we have introduced the parameters $A=2 G M /\left(H_{0}^{2} R_{0}^{3}\right)$ and $B=(1+$ $3 w) \Omega_{v}$. These equations were solved numerically by adopting the following procedure. For a given redshift, the initial value of the scale parameter is derived as well as the corresponding instant of time from the Hubble equation. If initially, at high redshifts (here taken around $z \sim 100$ ), the dark energy term is negligible, then using a Taylor expansion of the standard Lemaitre-Tolman solution, when the angle parameter $\theta<<1$ (see, for instance, Peebles 1980), the initial values of $y$ and its derivative $d y / d \tau$ can be estimated. For a given value of $w$, the parameter $A$ is varied until the condition defining the zerovelocity surface, e.g., $d y / d \tau=0$ at $y=1$ is satisfied. For the case $w=-2 / 3$, 
we have obtained $A=3.414$ and for the particular case $w=-1$, representing a cosmological constant, $A=3.658$. Therefore, the mass inside the zero-velocity radius $R_{0}$ is

$$
M=1.827 \frac{H_{0}^{2} R_{0}^{3}}{G}=4.1 \times 10^{12} h^{2} R_{0}^{3} M_{\odot}
$$

where $h=H_{0} /\left(100 \mathrm{kms}^{-1} M p c^{-1}\right)$ and $R_{0}$ is in Mpc. In the case $w=-2 / 3$, the numerical coefficient is slightly smaller (1.705 instead of 1.827). Comparing with eq. (1), we notice that the inclusion of the dark energy term represents, for a given $R_{0}$, an increase of about $28-38 \%$ on the mass derived by such a procedure.

Once the parameter $A$ is known, the velocity-distance relation, $\mathrm{v}=\mathrm{v}(\mathrm{R})$, for different shells at a given time is obtained by varying their energy. Shells with negative energy will expand, halt and fall back toward the center, while shells with positive energy expand forever, according to the aforementioned characterization of regions $i i$ and $i i i$. At a given time, there is a critical energy $E_{c}$ which defines the zero-velocity radius. Shells having $E<E_{c}$ have already crossed the turnaround point and are collapsing. Consequently, they have negative velocities. Shells with $E>E_{c}$ are still expanding and thus have positive velocities.

For the case $w=-1$, which gives a good representation of actual data, as we shall see in the next sections, the resulting numerical values are quite well fitted by the relation

$$
v(R)=-\frac{0.875 H_{0}}{R^{n}}\left(\frac{G M}{H_{0}^{2}}\right)^{(n+1) / 3}+1.274 H_{0} R
$$

with $n=0.865$. Notice that from the condition $v\left(R_{0}\right)=0$, eq. (6) is recovered. It is worth mentioning that solutions with negative energies are possible up to $R=2.30 R_{0}$, which defines the marginally bound surface. Beyond this critical radius, only unbound shells exist. The solution for $w=-2 / 3$ differs only marginally and will not be considered in further analyses.

For comparison, the velocity-distance relationship derived for the case $\Omega_{v}=0$ is

$$
v(R)=-1.038\left(\frac{G M}{R}\right)^{1 / 2}+1.196 H_{0} R
$$

which has a slightly flatter dependence on the distance than the precedent equation. 


\section{The Local Group}

An immediate application of the velocity-distance relationship is the determination of the Local Group mass, concentrated mainly on M31 and the Milky Way, as well as the Hubble parameter itself as we shall see later. Moreover, the velocity-distance relation gives also an indication of the dispersion of the peculiar velocities over the Hubble flow. As we have already mentioned, the local velocity dispersion is known to be quite small (Giraud 1986; Schlegel et al. 1994), a fact referred usually as the "coldness" of the local flow. An investigation of the dynamics of the Local Group and its environment by using numerical simulations was performed by Governato et al. (1997), who concluded that cold dark matter models $\left(\Omega_{m}=1\right.$ or $\left.\Omega_{m}=0.3\right)$ are unable to produce candidates embedded in regions having "cold" flows.

As a first step, we have searched to check the accuracy of eqs. (6) and (7) by using numerical simulations. We have performed N-body simulations using the adaptive particle-particle/particle-mesh $\left(A P^{2} M\right)$ code HYDRA (Couchman et al. 1995), with cosmological parameters $\mathrm{h}=0.65, \Omega_{m}=0.3, \Omega_{v}=0.7$ and $\sigma_{8}=0.9$. The simulation was performed in a periodic box of side $30 h^{-1}$ Mpc including $256^{3}$ particles, corresponding to a mass resolution of $2.05 \times$ $10^{8} M_{\odot}$. The simulation started at $\mathrm{z}=49$ and ended at the present time. Halos were initially detected by using a friends-of-friends (FOF) algorithm and, in a second step, unbound particles were removed by an iterative procedure. Thus, all halos in our catalog are gravitationally bound objects. For further details, the reader is referred to Peirani et al. (2004).

In our mock halo catalog, several examples of pairs with physical characteristics similar to the MW-M31 pair can be found. However, most of them have nearby (within 3-5 Mpc) halos of comparable mass or even higher, which perturb considerably the velocity field. Here, for illustration purposes only, we consider one case in which the main halos have masses respectively equal to $9.85 \times 10^{11}$ and $6.48 \times 10^{11}$ solar masses and are separated by a distance of $0.69 \mathrm{Mpc}$, parameters comparable to those of the M31-MW pair. About 73 subhalos were detected within $3 \mathrm{Mpc}$ of this pair, but satellites situated at distances larger than $2 \mathrm{Mpc}$ are clearly perturbed by other nearby structures, since the velocity dispersion increases considerably at those distances. Velocities and distances with respect to the center of mass were computed for all these objects.

A simple fitting of these simulated data gives $R_{0}=0.98 \pm 0.20 \mathrm{Mpc}$ and using eq. (6) with $\mathrm{h}=0.65$ (adopted in the simulations) a total mass of $(1.60 \pm 0.32) \times 10^{12} M_{\odot}$ is obtained. In spite of the excellent agreement with the actual total mass of the pair, this result is somewhat fortuitous since the velocity dispersion of the simulated satellites is rather high. In practice, besides 


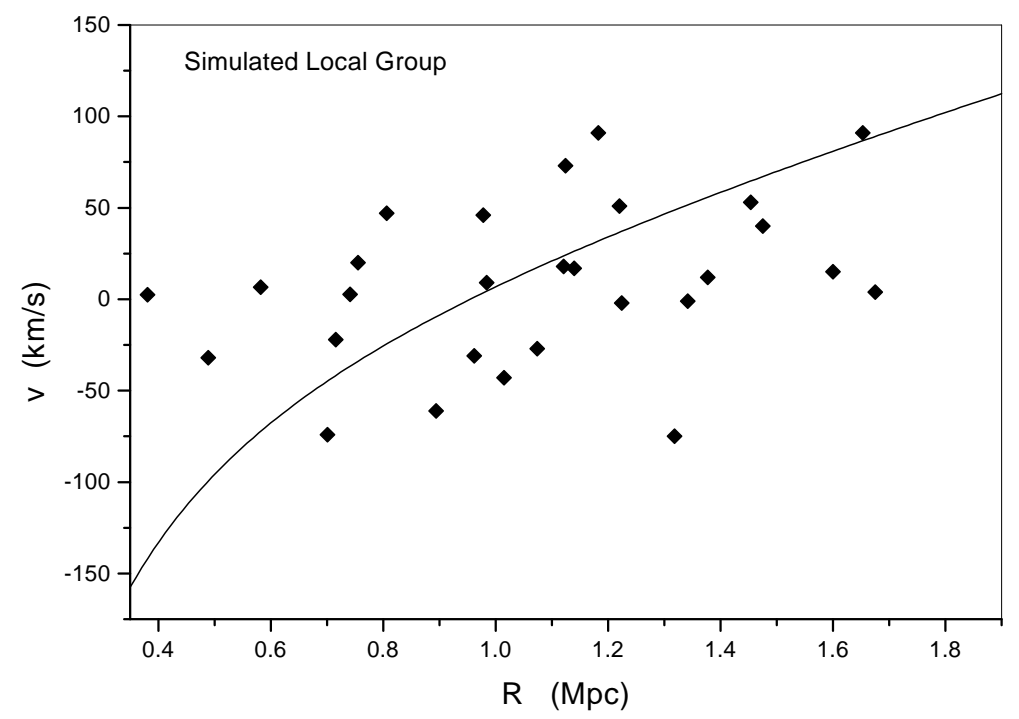

Fig. 1. Simulated velocity-distance data (diamonds) and best fit to the $\mathrm{v}=\mathrm{v}(\mathrm{R})$ relation (solid curve), corresponding to $M=1.48 \times 10^{12} M_{\odot}$ and $\sigma=73 \mathrm{~km} / \mathrm{s}$. The Hubble parameter was held constant, $\mathrm{h}=0.65$.

random motions, errors in distances or velocities increase considerably the uncertainty in the determination of the zero-velocity radius. These difficulties can be alleviated by searching the best fit of the $\mathrm{v}=\mathrm{v}(\mathrm{R})$ relation to data. By varying the mass in order to minimize the velocity dispersion and giving a higher weight to the inner satellites, one obtains $M=(1.48 \pm 0.30) \times 10^{12} M_{\odot}$. This result seems to be more confident to evaluate the uncertainties of the method. It is worth mentioning that quoted errors are estimates based on the spread of values derived from the fitting procedure and not formal statistical errors. In figure 1 we show the simulated velocity-distance data for satellites with $R \leq 1.8 \mathrm{Mpc}$ and the best fit solution for eq. (7). The derived 1-D velocity dispersion for this simulated data is $73 \mathrm{kms}^{-1}$, a value lower than that derived by Governato et al. (1997) but in quite good agreement with the $\Lambda$ CDM simulations by Macciò et al. (2005), who obtained a velocity dispersion of about $80 \mathrm{kms}^{-1}$ within a sphere of $3 \mathrm{Mpc}$ radius (see their Fig. 3). A flat, $\Lambda$ dominated cosmology is able to produce flows on scales of few Mpc around field galaxies "colder" than pure dark matter models, but somewhat higher than values derived from actual data, as we shall see below.

Recent data on neighboring galaxies of the Local Group were summarized by Karachentsev et al. (2002), who have estimated $R_{0}=0.94 \pm 0.10$ and derived from eq. (1) a total mass of $1.3 \times 10^{12} M_{\odot}$ for the M31/MW pair.

Here, eq. (7) was fitted to the data by Karachentsev et al. (2002), but varying 


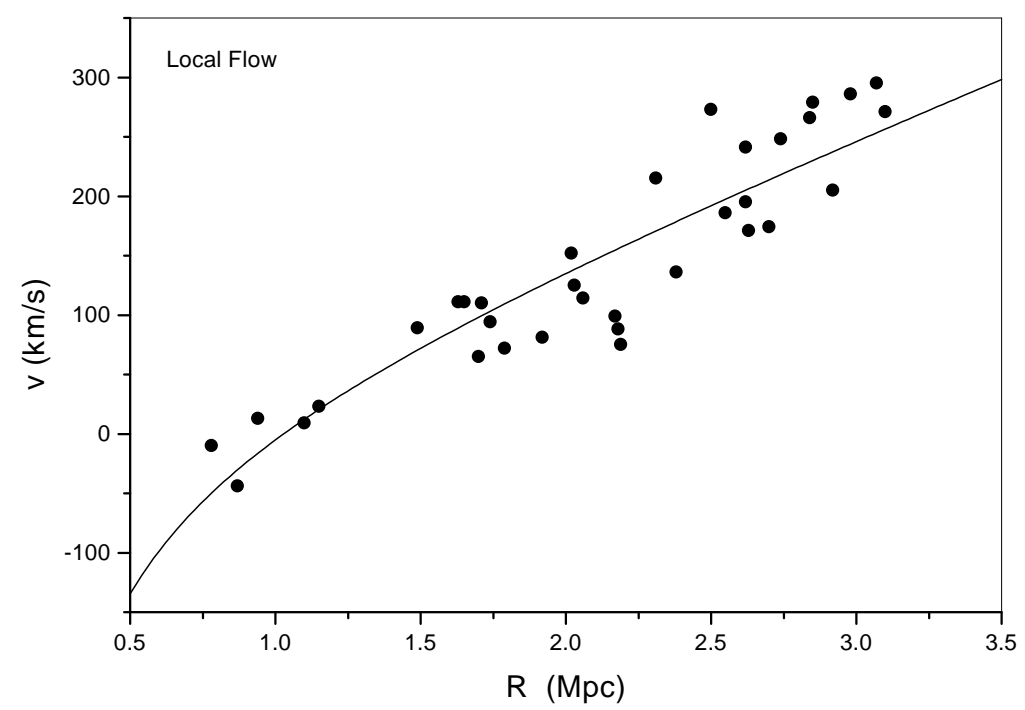

Fig. 2. Velocity and distance data (circles) for satellites of the M31-MW system (Karachentsev et al. 2002) and the best fit to the $\mathrm{v}=\mathrm{v}(\mathrm{R})$ relation, corresponding to $M=2.5 \times 10^{12} M_{\odot}$ and $\mathrm{h}=0.74$

now both the mass and the Hubble parameter in order to minimize the velocity dispersion. We have obtained $h=0.74 \pm 0.04$ and $M=(2.5 \pm 0.7) \times 10^{12} M_{\odot}$, where the quoted errors are again estimates based on the uncertainties of the fitted parameters. Figure 2 shows data points and the velocity-distance relation defined by the previous parameters. Had we used eq. (8) instead of eq. (7) in the fitting procedure, a similar result for the mass would have been obtained, but with a higher Hubble parameter, e.g., $h=0.87 \pm 0.05$. We shall return to this point latter.

The zero-velocity radius is located at $1.0 \pm 0.1 \mathrm{Mpc}$, which is about $10 \%$ higher than the value estimated by Karachentsev et al. (2002) and the "marginally" bound surface (zero-energy) is at a distance of about 2.3 Mpc. From our fit it results a 1-D velocity dispersion of $39 \mathrm{kms}^{-1}$, which should be compared with the value of $73 \mathrm{~km} / \mathrm{s}$ found from our simulated data for a similar scale. Macciò et al. (2005) have also revisited the "coldness" of the Hubble flow and, according to their final results, within a sphere of radius $3 \mathrm{Mpc}$, the expected velocity dispersion is $38 \mathrm{kms}^{-1}$, in rather good agreement with our figure. 


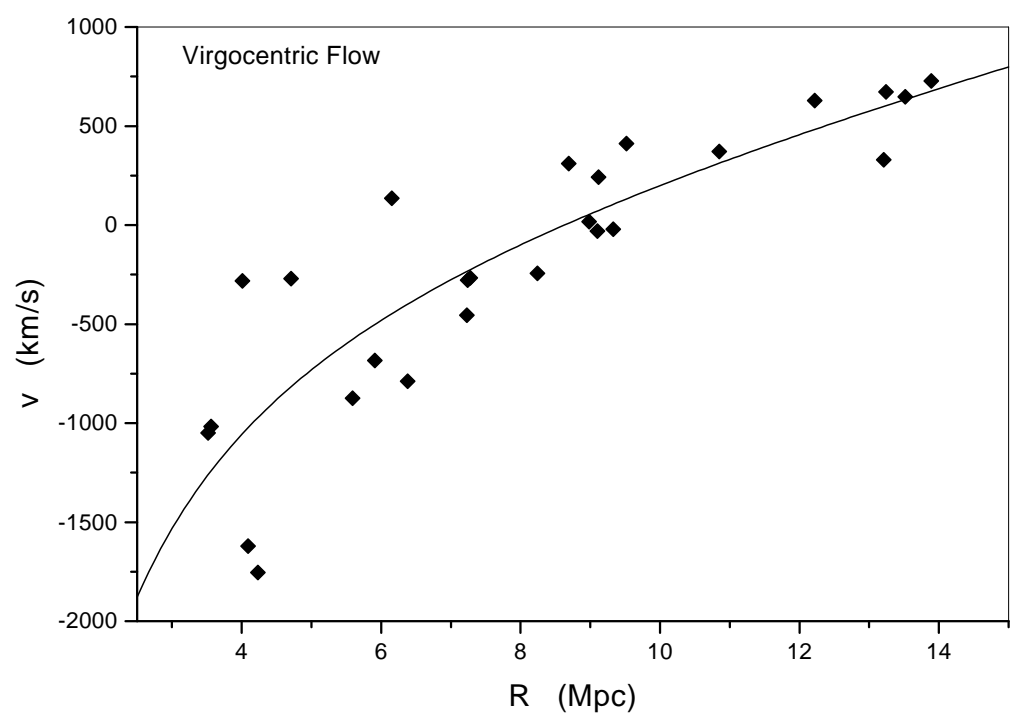

Fig. 3. Velocity and distance data for galaxies with Virgocentric distances in the range $3.5 \leq R \leq 15 \mathrm{Mpc}$ and the best fit to the $\mathrm{v}=\mathrm{v}(\mathrm{R})$ relation, for $M=1.1 \times 10^{15} M_{\odot}$ and $\mathrm{h}=0.65$

\section{The Virgo Cluster}

Dynamical models for the Virgo cluster based on the Lemaitre-Tolman model were, for instance, developed by Hoffman et al. (1980). They have modeled the projected velocity dispersion as a function of the angular distance and, from comparison with data, derived a mass of $(4.0 \pm 1.0) \times 10^{14} h^{-1} M_{\odot}$ contained inside a sphere of $6^{o}$ radius, which corresponds approximately to the central relaxed core of the cluster. Using the virial relation, Tully \& Shaya (1984) obtained a mass of $(7.5 \pm 1.5) \times 10^{14} M_{\odot}$ for this central core. More recently, Fouqué et al. (2001) using the Lemaitre-Tolman model derived a mass of $1.3 \times 10^{15} M_{\odot}$ inside a radius of $8^{\circ}$.

In this section, the derived velocity-distance relation (eq. 7), including effects of the cosmological constant, will be applied to galaxies outside the inner core of the Virgo cluster.

Galaxies with Virgocentric distances higher than $1.7 \mathrm{Mpc}$, corresponding approximately to the core radius, and less than $15 \mathrm{Mpc}$, were selected from the list by Teerikorpi et al. (1992), constituting a sub-sample of 27 objects. Distances derived from the Tully-Fisher relation were taken from the aforementioned source. Heliocentric velocities were corrected with respect to the Local Group according to the prescription by Courteau and van den Bergh 
(1999). Velocities and distances with respect to Virgo center were calculated by assuming a distance to the cluster of $16.8 \mathrm{Mpc}$ (Tully \& Shaya 1984) and an observed velocity of $967 \mathrm{~km} / \mathrm{s}$ (Kraan-Korteweg 1981).

The best fit of eq. (7) to data gives $h=0.65 \pm 0.09$ and $M=(1.10 \pm 0.12) \times$ $10^{15} M_{\odot}$, corresponding to a velocity dispersion $\sigma_{1 D}=335 \mathrm{~km} / \mathrm{s}$ for the flow. Figure 3 shows the velocity-distance data for the galaxies of our sample and the theoretical $\mathrm{v}=\mathrm{v}(\mathrm{R})$ relation computed with the derived parameters. The higher mass derived in the present analysis confirms some early results based on models of the velocity field in the vicinity of the Virgo cluster using the Lemaitre-Tolman equations, as those performed by Tully \& Shaya (1998) and Fouqué et al. (2001).

The resulting zero-velocity surface is located at $R_{0}=8.6 \pm 0.8 \mathrm{Mpc}$, which at the assumed distance corresponds to an angle $\theta_{Z V}=29^{\circ}$. This should be compared with the analyses by Hoffman et al. (1980), Tully \& Shaya (1984) or Teerikorpi et al. (1992), who estimated $\theta_{Z V}=27^{\circ}, 28^{\circ}$ and $25.8^{\circ}$ respectively.

Eq. (7), with the above parameters, predicts that at the level of the Local Group the observed velocity should be $988 \mathrm{~km} / \mathrm{s}$, which compares quite well with the observed value. This indicates that the projection of the peculiar velocity of the Local Group in the Virgo direction is about $190 \mathrm{~km} / \mathrm{s}$, compatible with the values found by de Freitas Pacheco (1985), Tamman \& Sandage (1985) and Federspiel et al. (1998). The marginally bound surface is located at about $19.8 \mathrm{Mpc}$, implying that the Local Group is bound to the cluster.

\section{Conclusions}

The contribution of a dark energy term in the mass-energy budget of the universe seems to be well established at the present time. In this study, the usual velocity-distance relation based on the Lemaitre-Tolman model, was revisited in order to include effects due to such a cosmological term.

The dynamical equations were solved numerically and the relation $M=$ $M\left(H_{0}, R_{0}\right)$, defining the mass inside the zero-velocity surface was recalculated. For a given $R_{0}$, the resulting masses are about $35-38 \%$ higher with respect to the original relation derived from the Lemaitre-Bondi model $\left(\Omega_{v}=0\right)$, if the dark energy is modeled by a fluid with an equation of state $P / \epsilon=w=-2 / 3$ or by a cosmological constant respectively.

The resulting $\mathrm{v}=\mathrm{v}(\mathrm{R})$ relation $(w=-1$ case $)$ was applied to the Local Group and to the Virgo cluster. From the best fitting procedure, a mass of $(2.5 \pm 0.7) \times$ $10^{12} M_{\odot}$ was derived for the M31-MW system, which corresponds to a M/L 
ratio of about $25 M_{\odot} / L_{B, \odot}$. The zero-velocity surface and the "marginally" bound surface are located at about $1.0 \pm 0.1 \mathrm{Mpc}$ and 2.3 Mpc respectively. The later defines in fact the boundaries of the Local Group.

More sophisticated analyses of the Local Group based on the action principle were performed by Peebles (1990). In this method, orbits are reconstructed in order to reproduce the observed radial velocities, maintaining the action stationary. Peebles (1990) preferred solution implies a mass of $6.4 \times 10^{12} M_{\odot}$ for the Local Group, a factor 2.5 higher than our result, but a comparison of these results could be meaningless since a different cosmology $(\Omega=0.1)$ was adopted in that work.

The same procedure applied to the Virgo cluster gives a mass of about $(1.10 \pm$ $0.12) \times 10^{15} M_{\odot}$ inside a radius of $8.6 \pm 0.8 \mathrm{Mpc}$. This mass is higher than the virial value (Tully \& Shaya 1984) or values derived from velocity dispersion profiles computed from models based on the Lemaitre-Tolman model (Hoffman et al. 1980). However, recent studies of the velocity field based on the Lemaitre-Tolman formulation also lead to masses compatible with our result. Our velocity-distance relation predicts a peculiar velocity of the Local Group towards the Virgo cluster of about $190 \mathrm{~km} / \mathrm{s}$, compatible with different estimates. The "marginally" bound surface encloses the Local Group, which in the future may attain its zero-velocity surface and then fall onto the cluster. However this prediction is based on a model in which the Local Group is considered as an "isolated" system. In reality the motion of the Local Group is much more complex, being strongly affected by large mass concentrations in the direction of Hydra-Centaurus (Great Attractor) and the Shapley supercluster. Some numerical simulations predict that within $\sim 30$ Gyr the Local Group will get closer to the Virgo center (in comoving coordinates), but then will be pulled away(in physical coordinates) due to the accelerated expansion of the Universe (Nagamine \& Loeb 2003).

The introduction of a cosmological constant term modifies the velocity-distance relation in comparison with that derived from the Lemaitre-Tolman model. Nevertheless both descriptions of the velocity field near the Local Group or the Virgo cluster yield masses comparable to within a factor of two. This is probably due to the fact that errors still present in distance estimates mask differences between both models. However, when searching for a best fit of both models to data, there is a substantial difference in the resulting Hubble parameter. The Lemaitre-Tolman model requires $\mathrm{h}$ in the range 0.87-0.92 in order to fit adequately the Local Group and Virgo data respectively, whereas eq. (7) requires $\mathrm{h}$ in the range $0.65-0.74$, more consistent with recent determinations and with the "concordant" model. In this sense, the inclusion of the cosmological constant in the $\mathrm{v}(\mathrm{R})$ relation seems to improve the representation of actual data. 


\section{Acknowledgement}

We thanks the anonymous referee for his useful comments. S. Peirani acknowledges the University of Nice-Sophia Antipolis for the financial support.

\section{References}

[1] Chernin, A., 2001, Physics Usp. 44, 1099

[2] Couchman, H.M.P., Thomas, P.A. and Pierce, F.R., 1995, ApJ, 452, 797

[3] Courteau, S. and van den Bergh, S., 1999, AJ 118, 337

[4] de Freitas Pacheco, J.A., 1985, AJ 90, 1007

[5] Federspiel, M., Tammann, G.A. and Sandage, A., 1998, ApJ 495, 115

[6] Fouqué, P., Solanes, J.M., Sanchis, T. and Balkowski, C., 2001, A\&A, 375, 770

[7] Giraud, E., 1986, A\&A 170, 1

[8] Governato, F., Moore, B., Cen, R., Stadel, J., Lake, G. and Quinn, T., 1997, NewA, 2, 91

[9] Hoffman, G.L., Olson, D.W. and Salpeter, E.E., 1980, ApJ 242, 861

[10] Huchra, J.P. and Geller, M.J., 1982, ApJ 257, 423

[11] Karachentseva, V.E. and Karachentsev, I.D., 1998, A\&A 127, 409

[12] Karachentseva, V.E. and Karachentsev, I.D., 2000, A\&AS 146, 359

[13] Karachentsev, I.D., Sharina, M.E., Makarov, D.I., Dolphin, A.E., Grebel, E.K., Geisler, D., Guhathakurta, P., Hodge, P.W., Karachentseva, V.E., Sarajedini, A. and Seitzer, P., 2002, A\&A 389, 812

[14] Karachentsev, I.D., 2005, AJ 129, 178

[15] Kilborn, V.A. et al., 2002, AJ 124, 690

[16] Kraan-Korteweg, R.C., 1981, A\&A 104, 280

[17] Lemaitre, G., 1933, Ann. Soc. Sci. Bruxelles, A53, 51

[18] Lynden-Bell, D., 1981, The Observatory 101, 111

[19] Macciò, A.V., Governato, F., and Horellou, C., 2005, MNRAS, astro-ph/0512583

[20] Nagamine, K. and Loeb, A., 2003, NewA, 8, 439

[21] Olson D.W. and Silk J., 1979, ApJ, 233, 395 
[22] Peebles, P.J.E., 1980, in The Large-Scale Structure of the Universe, Princeton Series in Physics, Princeton University Press, New Jersey, p. 80

[23] Peebles, P.J.E., 1990, ApJ, 362, 1

[24] Peirani, S., Mohayaee, R. and de Freitas Pacheco, J.A., 2004, MNRAS, 348, 921

[25] Perlmutter, S. et al.,1999, ApJ, 517, 565

[26] Riess, A.G. et al., 1998, AJ, 116, 1009

[27] Sandage, A., 1986, ApJ, 307, 1

[28] Schlegel, D., Davis, M. and Summers, F.J., 1994, ApJ, 427, 527

[29] Spergel, D.N. et al., 2003, ApJS, 148, 175

[30] Tammann, G.A. and Sandage, A., 1985, ApJ, 294, 81

[31] Teerikorpi, P., Bottinelli, L., Gouguenheim, L. and Paturel, G., 1992, A\&A, 260, 17

[32] Teerikorpi, P., Chernin, A.D. and Baryshev, Yu.V., 2005, A\&A submitted, astro-ph/0506683

[33] Tolman, R.C., 1934, Proc. Nat. Acad. Sci. 20, 169

[34] Tully, R.B. and Shaya, E.J., 1984, ApJ, 281, 31

[35] Tully, R.B. and Shaya, E.J., 1998, in Evolution of the Large Scale Structure, eds. R.F. Stein and A.G.W. Cameron, ESO, Garching, p. 333 